\title{
How Mild Traumatic Brain Injury May Affect Declarative Memory Performance in the Post-Acute Stage
}

\author{
Maja Stulemeijer, Pieter E. Vos, ${ }^{2}$ Sieberen van der Werf, Gert van Dijk, ${ }^{3}$ \\ Mark Rijpkema, ${ }^{4}$ and Guillén Fernández ${ }^{2,4}$
}

\begin{abstract}
Memory deficits are among the most frequently reported sequelae of mild traumatic brain injury (MTBI), especially early after injury. To date, these cognitive deficits remain poorly understood, as in most patients the brain is macroscopically intact. To identify the mechanism by which MTBI causes declarative memory impairments, we probed the functionality of the medial temporal lobe (MTL) and the prefrontal cortex (PFC), within 6 weeks after injury in 43 patients from a consecutive cohort, and matched healthy controls. In addition to neuropsychological measures of declarative memory and other cognitive domains, all subjects underwent functional magnetic resonance imaging (fMRI). Behavioral results showed poorer declarative memory performance in patients than controls, and decreasing performance with increasing duration of post-traumatic amnesia (a measure of injury severity). Task performance in the scanner was, as intended by the task and design, similar in patients and controls, and did not relate to injury severity. The task used reliably activated the MTL and PFC. Although we did not find significant differences in brain activity when comparing patients and controls, we revealed, in agreement with our neuropsychological findings, an inverse correlation between MTL activity and injury severity. In contrast, no difference in prefrontal activation was found between patients and controls, nor was there a relation with injury severity. On a behavioral level, injury severity was inversely related to declarative memory performance. In all, these findings suggest that reduced medial temporal functionality may contribute to poorer declarative memory performance in the post-acute stage of MTBI, especially in patients with longer post-traumatic amnesia.
\end{abstract}

Key words: functional magnetic resonance imaging; memory; mild brain concussion; neuropsychological tests

\section{Introduction}

$\mathbf{W}$ ITH AN INCIDENCE OF 100-300 PER 100,000 POPULATION, mild traumatic brain injury (MTBI) is one of the most prevalent neurological conditions worldwide (Cassidy et al., 2004). It is well recognized that memory deficits are common early after injury, and disabling complaints like forgetfulness and learning problems persist for months to years after injury in a minority of patients (Bohnen et al., 1994; Stulemeijer et al., 2007; van der Naalt et al., 1999). The mechanisms underlying these memory problems after MTBI remain poorly understood. Macroscopic abnormalities of brain tissue are often absent, and if present, do not consistently relate to cognitive impairments (Hofman et al., 2001; Levin et al, 1992; McCullagh et al., 2001; Sadowski-Cron et al., 2006). Rather, it has been hypothesized that functional alterations in brain structures involved in memory may underlie the deficits and complaints present after MTBI, especially early after injury (McAllister et al., 2001; Shaw, 2002; Umile et al., 2002). In the present study, we will investigate functional MRI correlates of declarative memory impairments, and seek to identify possible anatomic substrates for such impairments in patients who recently suffered an MTBI. In particular, we will probe the functionality of the medial temporal lobe (MTL) and the prefrontal cortex (PFC). Both brain regions play a critical role in learning and memory (Fernandez and Tendolkar, 2001; Squire and Zola-Morgan, 1991), and are known to be vulnerable to traumatic impact, either due to their anatomical location, or on the basis of deafferentation or cytotoxic/ excitotoxic processes (Bigler, 2007; Meythaler et al., 2001).

\footnotetext{
${ }^{1}$ Department of Medical Psychology, and ${ }^{2}$ Department of Neurology, Radboud University Nijmegen Medical Centre, Nijmegen, The Netherlands.

${ }^{3}$ Department of Neurology, Canisius Wilhelmina Hospital, Nijmegen, The Netherlands.

${ }^{4}$ Donders Institute for Brain, Cognition and Behaviour, Radboud University Nijmegen, Nijmegen, The Netherlands.
} 
Hence, we hypothesize that dysfunction of these regions may cause the memory deficits experienced by many patients early after MTBI.

Although research on this topic is scarce, several studies in both animals and humans provide indirect support for this hypothesis. In rodents, for example, MTBI-like injuries can produce memory impairments and dysfunction of the hippocampus, even without actual cell loss (Lowenstein et al., 1992; Lyeth et al., 1990; Slemmer et al., 2002). Similarly, a study in humans found significant associations between abnormal activity over scalp areas covering the temporal lobe and memory performance with magnetoencephalography (Lewine et al., 2007). In addition, subtle changes in temporal lobe metabolism are detected with nuclear imaging techniques like positron emission tomography for up to 6 weeks after MTBI, even in the absence of macroscopic tissue damage, and after the resolution of post-traumatic amnesia (Bergsneider et al., 2001; Jacobs et al., 1994; Umile et al., 2002). However, the relationship between perfusion abnormalities and cognitive performance is inconsistent (Ichise et al., 1994; Kant et al., 1997). Besides MTL dysfunction, impaired functionality of the PFC may negatively influence memory abilities. The critical role of the PFC in working memory is well established. Several processes attributed to the working memory system are in turn relevant for declarative long-term memory formation in the MTL. For example, cognitive operations like keeping information organized, suppressing irrelevant information, and binding of information across different modalities, may support the formation of a unitary episode in declarative memory (see Fernandez and Tendolkar, 2001, for a review). To date, fMRI studies on the involvement of the PFC in cognitive problems after MTBI have yielded varying results. The unifying theme seems to be that MTBI can induce changes in the allocation of processing resources needed for working memory and related cognitive tasks. For example, some studies have showed increased activation in prefrontal areas during mental effort relative to controls, which may suggest that MTBI patients allocate additional cognitive resources in order to perform within normal limits (Hillary et al., 2006; Jantzen et al., 2004; McAllister et al., 2001; Smits et al., 2009). In addition, activation peaks outside the working memory network are frequently reported for patients, but not controls (including activation in parahippocampal regions) (Chen et al., 2007; Smits et al., 2009). In all, there is reason to suggest that changes in both MTL and PFC processing may underlie some of the cognitive problems observed early after MTBI. Still, current knowledge is limited, as most of the few neuroimaging studies in this population had relatively small sample sizes, were often performed in selected samples (e.g., patients with persisting symptoms only), and have predominantly focused on frontal rather than temporal brain structures.

In the present study, we will apply behavioral measures of declarative memory and attention, and a neurophysiological measure of regional brain functionality, in a large sample of MTBI patients $(n=43)$ in the post-acute stage ( $<6$ weeks), and in 20 healthy matched controls. In addition to simple comparisons between patients and control subjects, we will relate trauma severity to behavioral and neurophysiological data to establish a closer relationship between trauma and its effects on brain functionality. To activate the MTL and PFC, subjects performed the so-called n-back working memory task (Gevins and Cutillo, 1993). The brain network subserving the n-back task, in which each new stimulus in a long series must be compared to the one presented $n$ steps back in the series, is known to involve primarily neocortical regions, particularly the dorsolateral PFC (Callicott et al., 1999; Owen et al., 2005). In addition, this task also produces a robust activity decrease or reduced engagement of the MTL, including the hippocampus (Egan et al., 2003; Meyer-Lindenberg et al., 2001). As the n-back paradigm can be equally well executed by patients with impaired declarative memory performance and controls, thereby excluding the confounding effects of different levels of test performance on brain activation, it is particularly suitable for use early after MTBI (McAllister et al., 1999; Price and Friston, 1999).

\section{Methods}

\section{Definition of MTBI}

In accordance with the criteria of the European Federation of Neurological Societies (EFNS), MTBI was defined as a history of impact to the head with or without loss of consciousness $(\mathrm{LOC}) \leq 30 \mathrm{~min}$, with or without post-traumatic amnesia (PTA), and with a hospital admission Glasgow Coma Scale score (GCS) of 13-15 (Vos et al., 2002).

\section{Subjects and procedure}

All consecutive MTBI patients admitted to the emergency department (ED) of the Radboud University Nijmegen Medical Centre and the Canisius Wilhelmina Hospital Nijmegen between October 2004 and August 2006 were contacted early after injury with the request to complete a questionnaire on premorbid and current functioning. Patients were asked to participate in the MRI study when they were between 18 and 50 years of age, able to participate within 6 weeks after injury, had no contraindications to MRI scanning, and reported good preinjury health (defined as no medication use and no previous neurological/psychiatric conditions). Each assessment started with MRI scanning, followed after a 20-min break by a neuropsychological screening. A total of 50 MTBI patients participated in this study. Seven patients were omitted from the analyses for the following reasons: missing MRI data due to claustrophobia $(n=2)$ or scanning session too demanding $(n=2)$, non-adherence to task instructions $(n=2)$, and the incidental finding of non-traumatic brain abnormalities $(n=1)$. Thus the final sample consisted of $43 \mathrm{MTBI}$ patients (24 males and 19 females, age range 18-49 years, mean age 31.1 years). In addition, 20 healthy subjects (11 males and 19 females, age range 18-50 years, mean age 33.5 years), recruited via advertisements, also participated in the study. No significant differences were found between the groups for age $(F=.79, d f(1,62), p=0.375)$, and gender (chi-square $=.004$, $d f(1), p=0.812)$. Also, no significant differences were found regarding educational level (scored on a seven-point scale that best fits the Dutch educational system, ranging from $1=$ primary school to $7=$ college and university) between patients $($ mean $=5.4, \mathrm{SD}=1.5)$ and controls [mean $=4.9$, $\mathrm{SD}=1.7 ; F=1.14, d f(1,62), p=0.291]$. All subjects were righthanded. All subjects gave written informed consent as approved by the local ethics committee.

\section{Data acquisition}

Clinical patient characteristics. Data on acute TBI variables such as LOC and PTA were collected by the consulting 
resident of neurology on the ED as part of a standardized procedure (see Stulemeijer et al., 2007 for a detailed description). In the present study, the duration of PTA was included as a measure of injury severity (Ahmed et al., 2000; Russel and Smith, 1961; Von Wowern, 1966). The assessment of the presence and resolution of PTA included the testing of anterograde memory (using a three-word recall test) and orientation, and a behavioral screen for confusion and agitation. If PTA had already ended on admission, an estimate about the duration was made based on all information available (e.g., a patient's first memory after the accident or eyewitness reports). When PTA persisted in the ED, the patients were admitted to the hospital for further monitoring. As most MTBI patients suffer only a brief period of PTA (in the range of minutes), and the first systematic assessment of PTA generally takes place at the ED, the exact determination of PTA duration is often impossible. Therefore, for the present study we grouped the patients based on the estimation of PTA duration into three clinically-meaningful categories that can be distinguished with a much higher degree of certainty: (1) no PTA, (2) PTA 1-30 minutes, and (3) PTA $>30 \mathrm{~min}$. This subdivision has also been used in previous studies and guidelines (e.g., Cantu, 2001; Stulemeijer et al., 2007).

General injury severity was scored with the Abbreviated Injury Score (AIS)/Injury Severity Score (ISS; Baker et al., 1974). Post-acute functioning of the patients was assessed using the early questionnaire mentioned above. The severity of the experienced post-concussion symptoms (PCS) was measured with the Rivermead Post-Concussion Questionnaire (RPQ). Subjects were asked to rate how problematic, if at all, each of 16 common PCS were experienced compared with the situation before they sustained their head injury (King et al., 1995). Limitations in activities of daily living were assessed with the subscale "Physical functioning" of the Short Form-36 (SF-36; Aaronson et al., 1998), and levels of posttraumatic stress with the Impact of Events Scale (IES; Sundin and Horowitz, 2002).

Neuropsychological screening. A neuropsychological test battery was administered to all subjects, covering cognitive domains commonly affected early after MTBI. The screening included the Dutch version of the National Adult Reading Test (general intelligence) (Schmand et al., 1991), the Digit-Symbol subtest of the Wechsler Adult Intelligence ScaleIII (Wechsler, 1997a) (complex attention and visuo-motor speed), the Stroop Colour Word Interference Test (reading speed, nomination speed, and inhibitory control) (Stroop, 1935), the California Verbal Learning Test (verbal memory) (Delis et al., 1987), and the subtest Faces from the Wechsler Memory Scale (visual memory) (Wechsler, 1997b). To assess emotional distress, all subjects completed the Beck Depression Inventory for Primary Care (Beck et al., 1997), and the Spielberger State-Trait Anxiety Inventory (Spielberger, 1983). The items regarding state anxiety were completed at the start of the fMRI session.

fMRI n-back task. Subjects were presented with a pseudorandom series of single digits (1-9, white on a black background) viewed in central vision via a prismatic mirror fitted in a head coil. The task was a simple blocked design with two alternating conditions over the course of 10 cycles. During the 0-back condition (minimal working memory load), individuals were asked to decide whether the current digit was a "1." During the 2-back condition (moderate working memory load), the task was to decide whether the digit currently presented matched the digit that had been presented two back in the sequence. On seeing a match, participants were asked to press a response-box button with their right index finger. When there was no match, no response was required. Each block consisted of a series of 15 digits, and was preceded by a sign indicating which condition was up next. As a reminder, this sign stayed on the screen below the digits throughout the block. Each digit was presented for $400 \mathrm{msec}$, followed by a 1500-msec interval. During each block there was a possibility of one, two, or three matches. Overall, each condition contained $17 \%$ targets. The total time to complete the task was $10 \mathrm{~min}$. Participants rehearsed the tasks outside the scanner to ensure understanding of task demands.

MRI data acquisition. For fMRI we acquired $2^{*}$-weighted images using an echo-planar imaging (EPI) sequence (Sonata $1.5 \mathrm{~T}$, Siemens, 33 axial slices, ascending slice acquisition, volume repetition time $[T R]=2.27 \mathrm{sec}$, echo time $[\mathrm{TE}]=30 \mathrm{msec}$, matrix size $=64 \times 64$, flip-angle $=90^{\circ}$, slice thickness $=3.0 \mathrm{~mm}$, slice gap $=0.5 \mathrm{~mm}$, field of view $[\mathrm{FOV}]=224 \mathrm{~mm}$ ). For structural MRI, we acquired T1-weighted images using a magnetization-prepared, rapid acquisition gradient echo (MPRAGE) sequence (176 sagittal slices, volume $\mathrm{TR}=2.250 \mathrm{sec}, \mathrm{TE}=$ $3.93 \mathrm{msec}$, slice-matrix size $=256 \times 256$, flip-angle $=15^{\circ}$, slice thickness $=1 \mathrm{~mm}, \mathrm{FOV}=256 \mathrm{~mm}$ ).

\section{Data analysis}

Analysis of behavioral data. Statistical analyses were carried out using SPSS for Windows v. 12.0 (SPSS Inc., Chicago, IL). All effects were tested at the $p<0.05$ level (twotailed). Univariate analyses of variance were used to make group comparisons. Effect sizes (Cohen's $d$ ) were calculated using the procedure described by Zakzanis (Zakzanis, 2001). By convention, effect sizes about 0.20 are considered small, those around 0.50 as moderate, and those from 0.80 and up as large. Chi-square analyses were used in case of dichotomous variables. As described in the section on data acquisition, the impact of injury severity was assessed by comparing subgroups based on PTA duration. Considering the small numbers of subjects per subgroup, non-parametric Kruskal-Wallis tests were performed for these within-group analyses.

\section{Analysis of $\mathrm{fMRI}$ data}

Single-subject (first-level) analyses. Image preprocessing and statistical analysis was performed using SPM2 software (http://www.fil.ion.ucl.ac.uk). The functional EPI-BOLD (blood-oxygenation-level-dependent) images were realigned, and the subject-mean was co-registered with the corresponding structural MRI using mutual information optimization. The functional images were subsequently slice-time corrected, spatially normalized, and transformed into a common space, as defined by the SPM2 MNI T1 template, as well as spatially filtered by convolving the functional images with an isotropic 3D gaussian kernel $(10 \mathrm{~mm}$ full width at half maximum; FWHM). A general linear model analysis, modeling stimulus-related activation as a boxcar function convolved with the canonical hemodynamic 
response function, was used to create contrast images for each participant, summarizing differences between block types (0back minus 2-back/2-back minus 0-back), and these images were used to create group average SPM (t) maps that were threshold at $p<0.05$ family-wise error corrected (FWE). To regress out movement-related activations, the realignment parameters were added to the model as covariates. The data were high pass-filtered $(128 \mathrm{sec})$ to account for various low-frequency effects.

Between-group (second-level) comparisons. The singlesubject contrast images were then entered into second-level (random effects) analyses, consisting of ANOVAs comparing patients and controls. In addition, we performed a simple regression analysis examining the relationship between brain activation and PTA duration. Four categories ranging from no to long PTA duration were included as regressors ( $1=$ controls, $2=$ no PTA, $3=$ PTA $1-30 \mathrm{~min}$, and $4=$ PTA $>30 \mathrm{~min}$ ). All analyses were first performed within regions of interest (ROI). The MNI space ROI of the dorsolateral PFC covered Brodmann areas 9 and 46, and was made using the WFU Pick Atlas toolbox for SPM (Maldjian et al., 2003). The second ROI covered the MTL, using the mask described by Amunts and associates (Amunts et al., 2005). In these ROI analyses, local maximum test statistics were employed and thresholded at an FWE corrected $p$ value $<0.05$. Subsequently we performed an exploratory whole-brain analysis using a threshold of $p=0.001$ (uncorrected). Coordinates are maxima in a given cluster according to the standard MNI template implemented in SPM2.

\section{Results}

\section{Clinical patient characteristics}

Injury characteristics of the patients are shown in Table 1. Overall, the participating patients appear to represent a typical MTBI population; most patients suffered their injuries from traffic-related accidents or falls, and experienced only a brief period of LOC with a varying duration of PTA. Macroscopic abnormalities were detected in four patients $(9 \%)$, and all four received a Trauma Coma Data Bank score of 2, which represents minor abnormalities that do not require neurosurgical intervention (Marshall et al., 1991). At time of the MR assessment none of the patients was admitted to the hospital; however, all patients experienced post-concussion symptoms, and reported substantial limitations in daily functioning. PTA duration was significantly correlated with cognitive (shown in Fig. 2), but not emotional or physical, post-concussion symptoms. Furthermore, no association between PTA duration and age, gender, education level, the presence or location of CT abnormalities, or other early outcome variables was found (data can be provided on request).

\section{Neuropsychological screening}

As shown in Table 2, a main effect of group was found for the tests in the memory domain, with patients scoring significantly poorer on both a visual and a verbal test of declarative memory. Effect sizes were moderate to large. For performance on tests in the attentional domain, no effect of group was found for the Stroop test for measuring informa-

Table 1. Injury Characteristics of the 43 Participating MTBi Patients

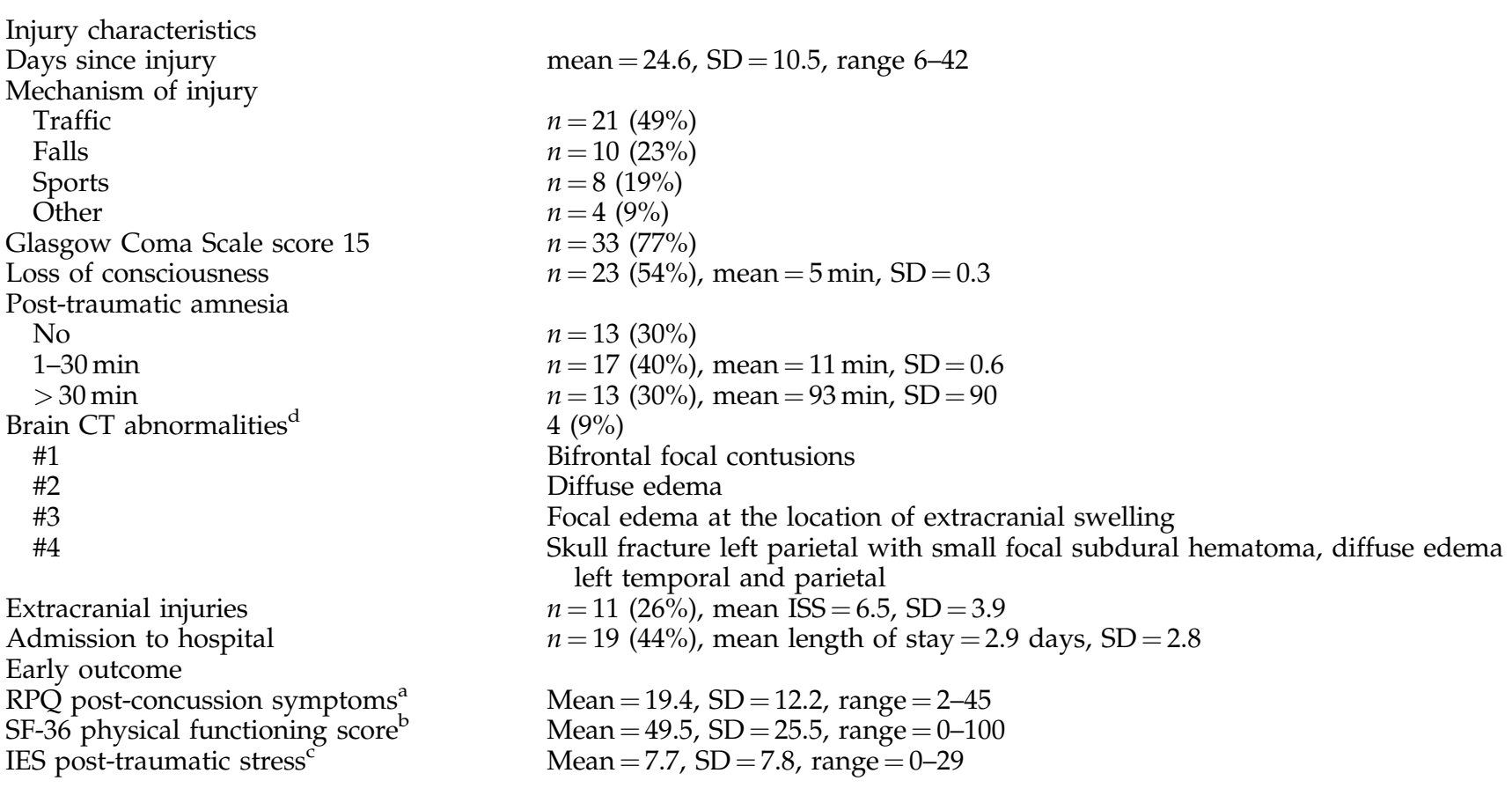

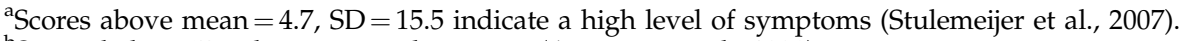

${ }^{\mathrm{b}}$ Scores below 65 indicate severe limitation (Aaronson et al., 1998).

'Scores above 26 indicate severe post-traumatic stress (Sundin and Horowitz, 2002).

${ }^{\mathrm{d} C} \mathrm{C}$ s were made according to published guidelines in 38 patients (Vos et al., 2002).

ISS, Injury Severity Score; RPQ, Rivermead Postconcussion Questionnaire; IES, Impact of Events Scale; SD, standard deviation; MTBI, mild traumatic brain injury; SF-36, Short Form-36. 
Table 2. N-Back Performance, Neuropsychological Test Scores, and Emotional Distress Scores For Patients AND CONTROls ${ }^{\mathrm{a}}$

\begin{tabular}{|c|c|c|c|c|c|c|}
\hline & \multicolumn{2}{|c|}{ Patients $(\mathrm{n}=43)$} & \multicolumn{2}{|c|}{ Controls $(\mathrm{n}=20)$} & \multirow[b]{2}{*}{ p Value } & \multirow[b]{2}{*}{ Effect size d } \\
\hline & Mean & $S D$ & Mean & $S D$ & & \\
\hline \multicolumn{7}{|l|}{ fMRI n-back performance } \\
\hline 0-back, $\%$ correct & 99.6 & 2.8 & 99.8 & 1.0 & .605 & 0.09 \\
\hline 0-back, mean rt (msec) & 497.0 & 68.6 & 484.2 & 61.4 & .517 & 0.19 \\
\hline 2-back, \% correct & 89.9 & 18.2 & 87.2 & 20.8 & .798 & 0.14 \\
\hline 2-back, mean rt (msec) & 620.1 & 118.2 & 594.8 & 92.8 & .527 & 0.23 \\
\hline \multicolumn{7}{|l|}{ Neuropsychological screening } \\
\hline \multicolumn{7}{|l|}{ Memory } \\
\hline \multicolumn{7}{|l|}{ Verbal memory (CVLT, z-scores) } \\
\hline Immediate recall (list A) & -1.1 & 2.2 & 0.3 & 2.1 & .011 & 0.65 \\
\hline Speed of learning & -1.6 & 2.1 & -0.3 & 3.0 & .034 & 0.55 \\
\hline Delayed-recall & -1.2 & 2.3 & 0.2 & 1.9 & .030 & 0.64 \\
\hline Recognition & 0.2 & 1.5 & -.6 & 1.8 & .080 & 0.50 \\
\hline Visual memory (WMS Faces, scaled score) & 6.9 & 3.6 & 10.40 & 4.4 & .002 & 0.91 \\
\hline \multicolumn{7}{|l|}{ Attention } \\
\hline $\begin{array}{l}\text { Complex attention (WAIS substitution, percentile) } \\
\text { Inhibitory control (Stroop, t-score) }\end{array}$ & 57.7 & 27.9 & 75.5 & 27.1 & .012 & 0.64 \\
\hline Card 1: reading speed & 49.9 & 8.5 & 50.6 & 13.5 & .501 & 0.07 \\
\hline Card 2: nomination speed & 51.2 & 9.1 & 55.4 & 10.1 & .130 & 0.45 \\
\hline Card 3: response inhibition & 53.4 & 8.0 & 57.1 & 10.6 & .091 & 0.42 \\
\hline$\Delta$ Card $3-$ card 2 & 53.7 & 7.4 & 55.1 & 9.9 & .912 & 0.17 \\
\hline \multicolumn{7}{|l|}{ Affective state } \\
\hline Depressed mood (BDI-PC) & 1.6 & 2.3 & 1.3 & 2.1 & .520 & 0.13 \\
\hline State anxiety $(\mathrm{STAI})^{\mathrm{b}}$ & 42.2 & 4.3 & 44.7 & 4.6 & .034 & 0.57 \\
\hline Trait anxiety (STAI) ${ }^{b}$ & 46.7 & 4.5 & 47.5 & 3.9 & .472 & 0.19 \\
\hline
\end{tabular}

an the case of the working memory task, raw scores are shown (as normative scores for fMRI tests are lacking). For other neuropsychological tests, we report the scaled scores in the output mode that the scoring system of each test provides.

${ }^{\mathrm{b}}$ Higher score is better (less anxiety).

NART, National Adult Reading Test; CVLT, California Verbal Learning Task; WMS, Wechsler Memory Scale; WAIS, Wechsler Adult Intelligence Scale; BDI-PC, Beck Depression Inventory for Primary Care; STAI, Spielberger State-Trait Anxiety Inventory; SD, standard deviation; fMRI, functional magnetic resonance imaging; IQ, intelligence quotient.

Group differences are tested with univariate analysis of variance.

tion processing speed (reading and naming), and executive control (color word interference). Patients did score significantly poorer on the WAIS symbol-digits test, which measures complex attention and visuo-motor speed, and which also has a memory component, depending on the strategy used (Joy et al., 2003). However, 39 patients (91\%) still performed within normal limits (defined as a score within 2 standard deviations of the appropriate age and gender population stratum). No difference was found between patients and controls in the level of estimated general intelligence. Regarding emotional distress, the patients were somewhat more anxious at time of the MRI assessment, but did not report higher levels of depression in the past weeks or trait anxiety. Significant correlations between test performance and PTA duration were found for the delayed-recall of verbal information (shown in Fig. 2), and complex attention, but not for the other tests or questionnaires (data can be provided on request).

\section{fMRI n-back performance}

As shown in Table 2, no significant differences were found between the patients and the controls regarding their performance on the n-back task, and effect sizes were small. Response times were significantly slower during the 2-back condition $[F(1,61)=94.8, p<0.001]$, but no effect of group was found $[F(1,61)=0.8, p=0.374]$. Similarly, more errors were made in the 2-back condition than the 0-back condition $[F(1,61)=20.2, p<0.001]$, but no differences were found between patients and controls $[F(1,61)=0.2, p=0.675]$. No significant differences in working memory performance were found between the three PTA duration categories. For example, mean response times in the 2-back condition were as follows: controls $=594.8$ (SD 92.8), no PTA $=587.6$ (SD 119.7), PTA $1-30 \mathrm{~min}=633.5(\mathrm{SD} \mathrm{100.7)}$, and PTA $>30 \mathrm{~min}=640.4$ (SD 145.4) [Kruskal-Wallis chi-square $(3,60)=2.8, p=0.411$ ]. Additionally, the mean percentages correct in the 2-back condition were as follows: controls $=87.2$ (SD 20.8), no PTA $=93.7$ (SD 7.2), PTA 1-30 $\mathrm{min}=84.1$ (SD 26.9), PTA $>30 \mathrm{~min}=87.3 \quad(\mathrm{SD} \quad 22.8) \quad$ [Kruskal-Wallis chi-square $(3,60)=0.8, p=0.842]$.

\section{Functional imaging results}

Main effects $n$-back task. The n-back task activated the expected network of brain areas (Owen et al., 2005). Thus, the 0 minus 2-back contrast showed activation in bilateral hippocampi, bilateral middle/inferior temporal gyri, bilateral superior medial gyri, bilateral rolandic operculi, and bilateral middle cingulate cortices. On the other hand, the 2 minus 
0-back contrast showed activation in the bilateral dorsolateral prefrontal cortices, bilateral middle/inferior frontal gyri, superior frontal cortices, bilateral insula, supplementary motor areas, bilateral inferior parietal areas, and the cerebellum bilaterally. Shown in Figure 1 is the activation related to the two task conditions in the MTBI patients. Comparable activations were found in controls [threshold $\mathrm{p}(\mathrm{FWE})<0.01$, extent $>25$ voxels (threshold used for visualization purposes)]. Figures and detailed coordinates are available on request.

Between-group differences. No significant differences in brain activation were found between patients and controls in either the PFC (2-back minus 0-back contrast), or the MTL region of interest (0-back minus 2-back contrast). Additionally, explorative whole-brain analyses did not reveal significant activation differences between groups in response to both task conditions in other brain areas. To examine the potential confounding effect of higher state anxiety and poorer attentional performance in patients, we exploratively repeated the second-level between-group analysis twice: once with the scores of the state anxiety questionnaire as a covariate, and once with the scores on the WAIS digit-symbol test of complex attention as a covariate. These re-analyses did not change the results.

Association with injury severity. A significant negative correlation between PTA duration and MTL activation in 0-back minus 2-back contrast was found in the left hippocampus [local maximum $\mathrm{x}=-16, \mathrm{y}=-30, \mathrm{z}=-18, \mathrm{p}(\mathrm{FWE})=$ $0.041, \mathrm{k}=52]$, and a trend was found in the right hippocampus [local maximum $\mathrm{x}=18, \mathrm{y}=-16, \mathrm{z}=-26, \mathrm{p}(\mathrm{FWE})=$ $0.075, \mathrm{k}=28$ ]. The fMRI and behavioral correlates of PTA duration are shown in Figure 2. In addition, explorative whole-brain analyses showed a significant negative correlation between PTA duration and a cluster of activation in the brainstem, encompassing the left and right pons (local maximum $\mathrm{x}=14, \mathrm{y}=-16, \mathrm{z}=-26, \mathrm{k}=807, p$ [uncorrected] $<0.001)$.

\section{Discussion}

In this study we used behavioral assessment and fMRI to investigate memory performance in a large sample of patients who recently suffered MTBI, and healthy controls. The MTBI patients performed poorer on a test of declarative memory, and this performance was proportionally related to the severity of injury. In addition, injury severity was related to more severe cognitive complaints. The fMRI results showed a negative correlation between injury severity and activation strength in the MTL. Together, these findings point toward a contribution of functional alterations of the MTL to cognitive dysfunction early after MTBI.

To our knowledge, our study is the first to explore MTL functionality in MTBI patients using fMRI. The results suggest that MTBI disrupts the normal MTL disengagement pattern during performance of the n-back working memory task, as longer PTA duration related to less activation in the MTL in the 0-back minus 2-back contrast. As discussed by others in different patient populations, this pattern likely reflects an impaired ability to suppress MTL activity during the performance of the 2-back condition (Egan et al., 2003; MeyerLindenberg et al., 2001; Pochon et al., 2002; Snaphaan et al., 2009). Insufficient suppression may cause incidental learning of the digits, thereby reducing the efficiency of working memory processes. Alternatively, due to the subtraction logic of our block design, our findings might also reflect more activation during the 2-back condition in relation to longer PTA. MTBI patients with more severe injuries may need additional MTL support in order to sustain the common working memory circuit. However, this latter explanation seems unlikely. In contrast to previous studies that found hippocampal activation during a working memory task, our paradigm is very simple, it does not require feature binding, and it contains only a very brief maintenance period (Olson et al., 2006; Piekema et al., 2006). Hence, MTBI severity seems to go along with functional changes in the MTL that might explain the effects of MTBI on declarative memory performance.

Although the 2-back condition strongly activated the expected regions associated with working memory (Owen et al., 2005), including the PFC, no activation differences were found between patients and controls in the frontal lobes, and prefrontal activation did not relate to injury severity. In addition, patients did not show signs of impairments on the Stroop Colour-Word task, which is considered sensitive for frontal dysfunction (Demakis, 2004). Hence, in contrast to previous
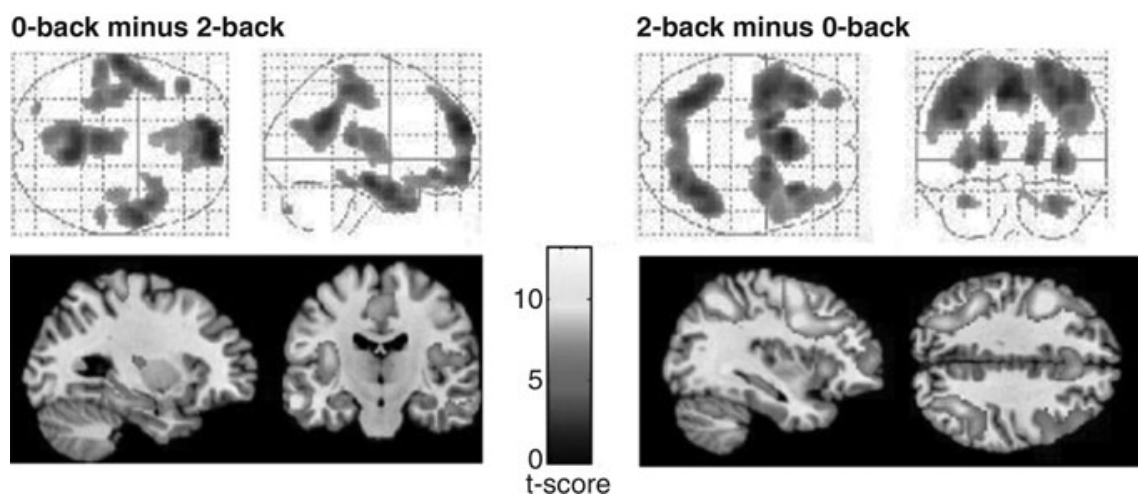

FIG. 1. Maximum-intensity projections of the statistical parametric maps (upper panels) of n-back task load comparisons in the patients, and activation maps of selected sagittal, coronal, and transverse slices (lower panel). Left: Areas of significantly higher activation in the 0-back minus 2-back contrast. Right: Areas of significantly higher activation in the 2-back minus 0back contrast. Displayed threshold $\mathrm{p}(\mathrm{FWE})<0.001$, extent $>25$ voxels. See the text for further details (FEW, family-wise error corrected). 


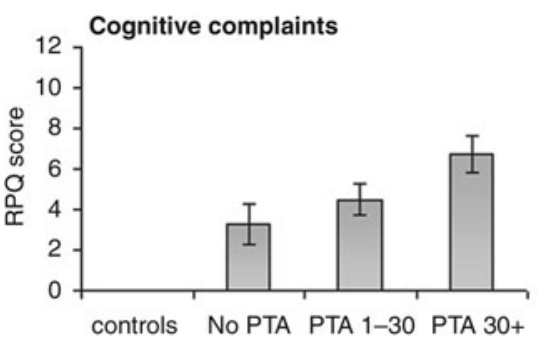

MTL activation
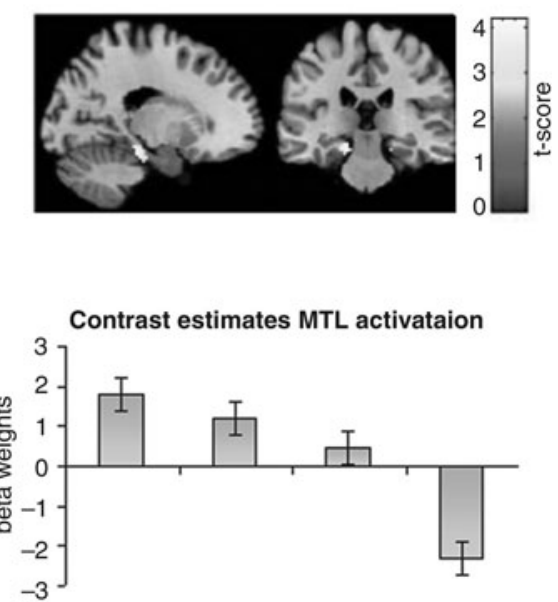

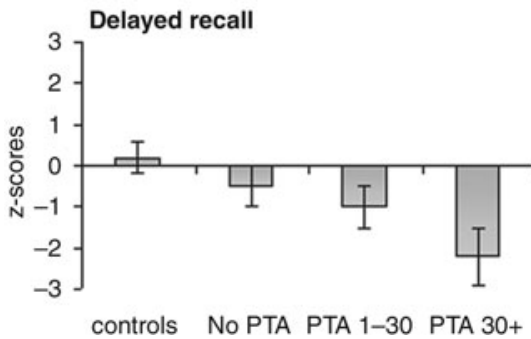

controls No PTA PTA 1-30 PTA $30+$

FIG. 2. Left: Severity of self-reported cognitive problems (upper panel), and performance on a declarative memory test (delayed-recall of the California Verbal Learning Test, lower panel). Values are displayed as means with standard error. Right: Region in the MTL that correlated with PTA duration [global maximum $x=-16, y=-30, z=-18, p(F W E)=0.041$, $\mathrm{k}=52$ ], derived from the second-level region of interest regression analysis, $p=0.001$ correction for spatial extent (upper panel). The beta weights were extracted from the between-groups ANOVA (most significant voxel in the MTL, lower panel) (RPQ, Rivermead Postconcussion Questionnaire, MTL, medial temporal lobe; PTA, post-traumatic amnesia; ANOVE, analysis of variance; FWE, family-wise error corrected).

studies, our findings do not seem to support the hypothesis that MTBI patients have to allocate additional compensatory efforts in order to obtain the same behavioral results (Jantzen et al., 2004; McAllister et al., 1999, 2001), nor that frontal dysfunction is present (Chen et al., 2007; Easdon et al., 2004). However, we cannot rule out MTBI effects of PFC functionality, as the n-back activation does not cover the entire PFC, and the design of our study does not allow exact replication of previous findings. For example, McAllister and coworkers found a disproportional increase of activation in the PFC under the 1-back versus 0 -back condition in MTBI patients, but less incremental activation under the 2-back versus 1-back condition (McAllister et al., 1999). As cerebral activation and increasing cognitive load may not be related in a linear manner, differences in activation between patients and controls might only become apparent when a parametric modulation is introduced (Callicott et al., 1999; McAllister et al., 1999). As reviewed by Hillary and associates, further characterization of the role of PFC in modulating working memory performance in clinical samples will require the use of longitudinal examinations, parametric manipulations with tight control over task load/performance relationships, and both verbal and nonverbal working memory paradigms (Hillary et al., 2006).

Results of the present study support previous work suggesting that dysfunction of the MTL might underlie the memory deficits commonly observed early after MTBI. Nevertheless, many questions remain about how MTBI induces these deficits, what magnitude of injury is required to trigger the dysfunction, and why the MTL would be especially vulnerable to damage. It seems unlikely that direct structural injury to the hippocampus accounts for most of the memory deficits seen in MTBI patients, because circumscribed microscopic lesions that might cause indirect trauma effects are rare. Rather, there is growing interest in the role of neurochemical alterations (e.g., in the cholinergic system) in the development of acute as well as late cognitive deficits (e.g., Arciniegas et al., 2003; Salmond et al., 2005). For example, patients with more severe TBI displayed atrophic changes of brain tissue that coincided with the distribution of the cholinergic system in the brain, including the bilateral hippocampus (Salmond et al., 2005). In case of MTBI, disruption of the cholinergic system may be transient in most patients, followed by recovery rather than cell loss. The presence of cholinergic dysfunction, its natural history, and its relationship with memory performance after MTBI, all need to be better established, as do post-TBI neurochemical alterations in other systems (e.g., glutamatergic, dopaminergic, and noradrenergic). Proper functioning of the MTL depends on intact network connections with many other brain areas, and memory functionality may also be comprised by disturbances in the axonal connections within or between these networks (Arcienigas et al., 2001). Several studies have found evidence for reduced axonal integrity in normal-appearing white matter in MTBI patients, hours to years after injury, by using diffusion tensor imaging (DTI). DTI quantifies white matter architecture through an extensive description of water diffusion in brain tissue (Belanger et al., 2007; Ulug et al., 1999). Affected brain structures commonly, but not consistently, include the corpus callosum and the internal capsule (Arfanakis et al., 2002; Bazarian et al., 2007; Inglese et al., 2005). Additionally, differences between MTBI patients and healthy controls have also occasionally been found in other brain structures, such as the fornix (connecting the hippocampus to the hypothalamus; Nakayam et al., 2006), and cingulum (connecting components of the limbic system Rutgers et al., 2008).

An unexpected yet intriguing finding was the strong negative correlation between the duration of PTA and pons 
activation in the 0-back minus 2-back contrast. This brainstem structure is part of the ascending reticular activating system, which governs overall basic arousal level, serves as a relay station for cerebellar afferents, and is functionally related to corticopontine projections in the parietal and prefrontal areas (Schmahmann and Pandya, 1997). The role of the pons in higher-order cognitive processes is not well-established, although pons activation during several difficult cognitive tasks (e.g., mental arithmetic [Critchley et al., 2000], and declarative memory [Weis et al., 2004; Chen and Desmond, 2005) has been observed. Nevertheless, previous work reported a correlation between delayed central brainstem conduction (brainstem evoked potentials) and PTA (Watson et al., 1995). In addition, studies using single-photon emission computed tomography have found relationships between the presence or duration of PTA and the degree of brainstem hypoperfusion (Gowda et al., 2006; Lorberboym et al., 2002), and brainstem auditory evoked potentials have been demonstrated to be abnormal in 10-40\% of patients with acute MTBI (Rizzo et al., 1983; Schoenhuber et al., 1983; Soustiel et al., 2005). Thus our results may reflect that even after PTA has resolved, the brainstem is still somewhat functionally impaired. Considering the lack of an a priori hypothesis regarding brainstem dysfunction, and the fact that the pons is not among the structures known to be necessary to perform the n-back task, nor is it among the specific brainstem areas most likely to be involved in the genesis of post-traumatic neurobehavioral disturbances, interpretation of these findings requires caution.

To put the results in perspective, several methodological limitations of our study should be taken into account. First, in the present study only patients without significant emotional or physical problems before the injury were included, and most participants had suffered "uncomplicated" MTBI (e.g., without CT evidence of abnormalities). This approach offers an uncomplicated view of the impact of MTBI on cognition, but one should be aware that this might limit the generalizability of the findings to the entire MTBI population. Furthermore, we used a 6 week post-injury interval, a period during which many changes may be occurring at different time points and to different degrees among patients. This may have created a large variance in the data, influencing the results to an unknown degree. Given the large inter-individual differences in recovery after MTBI, using a shorter post-injury interval will probably not solve this problem; rather, future researchers may consider performing repeated measurements within individuals to track the process of recovery. Last, although our study population was larger than those of most prior studies, the subgroups were still relatively small, resulting in decreased power to detect significant differences, especially since we applied a rather conservative correction method for most analyses.

There is considerable debate about the impact of MTBI on cognitive abilities. Generally, it is assumed that neurological/ somatic factors explain the acute disturbances, whereas psychological factors account for most of the late cognitive problems (Iverson, 2005). However, few studies have measured brain activation during cognitive efforts in the first weeks after injury, and integrated neurophysiological and behavioral findings. The results presented in this article contribute to this discussion by revealing indirect support for a system-level mechanism by which MTBI may cause a de- clarative memory deficit, even in the post-acute stage. The MTL (and possibly also the pons) may be especially relevant for understanding MTBI-induced changes in the brain, and could be selected as a region of interest in future analyses. The clinical utility (e.g., guiding management) of our findings is yet unknown. It is well known that cognitive deficits after MTBI resolve completely and spontaneously in most patients within 3 months, regardless of initial injury severity, whereas a minority will develop chronic cognitive sequelae. It is a challenge for future researchers to demonstrate that early MTL dysfunction has the power to identify patients at risk for such long-term cognitive problems. There is preliminary evidence that fMRI is a sensitive tool for investigating changes in brain functionality related to recovery after MTBI (Jantzen et al., 2004).

In conclusion, by using fMRI, we were able to detect alterations in functionality of the brain in patients with predominantly uncomplicated MTBI that would go unnoticed using standard imaging techniques. The findings point toward a role of reduced medial temporal lobe functionality in the causation of impaired declarative memory processing in the post-acute stage, especially in patients with more severe injury.

\section{Acknowledgments}

We would like to thank all subjects for their participation, and Jeske Broeren, Sanne Rasing, and Paul Gaalman for their assistance with the data acquisition. The Top Center Traumatology Nijmegen is kindly acknowledged for its financial support.

\section{Author Disclosure Statement}

All authors state that no competing financial interests exist.

\section{References}

Aaronson, N.K., Muller, M., Cohen, P.D., Essink-Bot, M.L., Fekkes, M., Sanderman, R., Sprangers, M.A., Velde, A.T., and Verrips, E. (1998). Translation, validation, and norming of the Dutch language version of the SF-36 Health Survey in community and chronic disease populations. J. Clin. Epidemiol. 51, 1055-1068.

Ahmed, S., Bierley, R., Sheikh, J.I., and Date, E.S. (2000). Posttraumatic amnesia after closed head injury: a review of the literature and some suggestions for further research. Brain Inj. 14, 765-780.

Amunts, K., Kedo, O., Kindler, M., Pieperhoff, P., Mohlberg, H., Shah, N.J., Habel, U., Schneider, F., and Zilles, K. (2005). Cytoarchitectonic mapping of the human amygdala, hippocampal region and entorhinal cortex: intersubject variability and probability maps. Anat. Embryol. 210, 343-352.

Arciniegas, D.B. (2003). The cholinergic hypothesis of cognitive impairment caused by traumatic brain injury. Curr. Psychiatry Rep. 5, 391-399.

Arciniegas, D.B., Topkoff, J.L., Rojas, D.C., Sheeder, J., Teale, P., Young, D.A., Sandberg, E., Reite, M.L., and Adler, L.E. (2001). Reduced hippocampal volume in association with p50 nonsuppression following traumatic brain injury. J. Neuropsychiatry Clin. Neurosci. 13, 213-221.

Arfanakis, K., Haughton, V.M., Carew, J.D., Rogers, B.P., Dempsey, R.J., and Meyerand, M.E. (2002). Diffusion tensor MR imaging in diffuse axonal injury. Am. J. Neuroradiol. 23, 794-802. 
Baker, S.P., Oneill, B., Haddon, W., and Long, W.B. (1974). Injury Severity Score-Method for describing patients with multiple injuries and evaluating emergency Care. J. Trauma 14, 187-196.

Bazarian, J.J., Zhong, J., Blyth, B., Zhu, T., Kavcic, V., and Peterson, D. (2007). Diffusion tensor imaging detects clinically important axonal damage after mild traumatic brain injury: a pilot study. J. Neurotrauma 24, 1447-1459.

Beck, A.T., Guth, D., Steer, R.A., and Ball, R. (1997). Screening for major depression disorders in medical inpatients with the Beck Depression Inventory for Primary Care. Behav. Res. Ther. 35, 785-791.

Belanger, H.G., Vanderploeg, R.D., Curtiss, G., and Warden, D.L. (2007). Recent neuroimaging techniques in mild traumatic brain injury. J. Neuropsychiatry Clin. Neurosci. 19, 5-20.

Bergsneider, M., Hovda, D.A., McArthur, D.L., Etchepare, M., Huang, S.C., Sehati, N., Satz, P., Phelps, M.E., and Becker, D.P. (2001). Metabolic recovery following human traumatic brain injury based on FDG-PET: time course and relationship to neurological disability. J. Head Trauma Rehabil. 16, 135-148.

Bigler, E.D. (2007). Anterior and middle cranial fossa in traumatic brain injury: Relevant neuroanatomy and neuropathology in the study of neuropsychological outcome. Neuropsychology 21, 515-531.

Bohnen, N., Van Zutphen, W., Twijnstra, A., Wijnen, G., Bongers, J., and Jolles, J. (1994). Late outcome of mild head injury: results from a controlled postal survey. Brain Inj. 8, 701-708.

Callicott, J.H., Mattay, V.S., Bertolino, A., Finn, K., Coppola, R., Frank, J.A., Goldberg, T.E., and Weinberger, D.R. (1999). Physiological characteristics of capacity constraints in working memory as revealed by functional MRI. Cerebral Cortex 9, 20-26.

Cantu, R.C. (2001). Posttraumatic retrograde and anterograde amnesia: Pathophysiology and implications in grading and safe return to play. J. Athl. Train. 36, 244-248.

Cassidy, J.D., Carroll, L.J., Peloso, P.M., Borg, J., Von Holst, H., Holm, L., Kraus, J., and Coronado, V.G. (2004). Incidence, risk factors and prevention of mild traumatic brain injury: results of the WHO Collaborating Centre Task Force on Mild Traumatic Brain Injury. J. Rehabil. Med. 43, 28-60.

Chen, J.K., Johnston, K.M., Collie, A., McCrory, P., and Ptito, A. (2007). A validation of the post concussion symptom scale in the assessment of complex concussion using cognitive testing and functional MRI. J. Neurol. Neurosurg. Psychiatry 78, 1231-1238.

Chen, S.H., and Desmond, J.E. (2005). Temporal dynamics of cerebro-cerebellar network recruitment during a cognitive task. Neuropsychologia 43, 1227-1237.

Critchley, H.D., Corfield, D.R., Chandler, M.P., Mathias, C.J., and Dolan, R.J. (2000). Cerebral correlates of autonomic cardiovascular arousal: a functional neuroimaging investigation in humans. J. Physiol. 523, 259-270.

Delis, D.C., Kramer, J.H., Kaplan, E., and Ober, B.A. (1987). California Verbal Learning Test. Harcourt Brace Jovanovich Inc.: New York.

Demakis, G.J. (2004). Frontal lobe damage and tests of executive processing: a meta-analysis of the category test, Stroop test, and trail-making test. J. Clin. Exp. Neuropsychol. 26, 441-450.

Easdon, C., Levine, B., O'Connor, C., Tisserand, D., and Hevenor, S. (2004). Neural activity associated with response inhibition following traumatic brain injury: an event-related fMRI investigation. Brain Cogn. 54, 136-138.

Egan, M.F., Kojima, M., Callicott, J.H., Goldberg, T.E., Kolachana, B.S., Bertolino, A., Zaitsev, E., Gold, B., Goldman, D.,
Dean, M., Lu, B., and Weinberger, D.R. (2003). The BDNF val66met polymorphism affects activity-dependent secretion of BDNF and human memory and hippocampal function. Cell $112,257-269$.

Fernandez, G., and Tendolkar, I. (2001). Integrated brain activity in medial temporal and prefrontal areas predicts subsequent memory performance: human declarative memory formation at the system level. Brain Res. Bull. 55, 1-9.

Gevins, A., and Cutillo, B. Spatiotemporal dynamics of component processes in human working memory. (1993). Electroencephalogr. Clin. Neurophysiol. 87, 128-143.

Gowda, N.K., Agrawal, D., Bal, C., Chandrashekar, N., Tripati, M., Bandopadhyaya, G.P., Malhotra, A., and Mahapatra, A.K. (2006). Technetium Tc-99m ethyl cysteinate dimer brain single-photon emission CT in mild traumatic brain injury: a prospective study. Am. J. Neuroradiol. 27, 447-451.

Hillary, F.G., Genova, H.M., Chiaravalloti, N.D., Rypma, B., and DeLuca, J. (2006). Prefrontal modulation of working memory performance in brain injury and disease. Hum. Brain Mapp. 27, 837-847.

Hofman, P.A.M., Stapert, S.Z., Van Kroonenburgh, M.J.P.G., Jolles, J., De Kruijk, J., and Wilmink, J.T. (2001). MR imaging, single-photon emission $\mathrm{CT}$, and neurocognitive performance after mild traumatic brain injury. Am. J. Neuroradiol. 22, 441-449.

Ichise, M., Chung, D.G., Wang, P., Wortzman, G., Gray, B., and Franks, W. (1994). Technetium-99M-Hmpao SPECT, CT, and MRI in the evaluation of patients with chronic traumatic brain injury-A correlation with neuropsychological performance. J. Nucl. Med. 35, 217-226.

Inglese, M., Makani, S., Johnson, G., Cohen, B.A., Silver, J.A., Gonen, O., and Grossman, R.I. (2005). Diffuse axonal injury in mild traumatic brain injury: a diffusion tensor imaging study. J. Neurosurg. 103, 298-303.

Iverson, G.L. (2005). Outcome from mild traumatic brain injury. Curr. Opin. Psychiatry 18, 301-317.

Jacobs, A., Put, E., Ingels, M., and Bossuyt, A. (1994). Prospective evaluation of technetium-99m-HMPAO SPECT in mild and moderate traumatic brain injury. J. Nucl. Med. 35, 942-947.

Jantzen, K.J., Anderson, B., Steinberg, F.L., and Kelso, J.A.S. (2004). A prospective functional MR imaging study of mild traumatic brain injury in college football players. Am. J. Neuroradiol. 25, 738-745.

Joy, S., Fein, D., and Kaplan, E. (2003). Decoding digit symbol: speed, memory, and visual scanning. Assessment 10, 56-65.

Kant, R., Smith-Seemiller, L., Isaac, G., and Duffy, J. (1997). TcHMPAO SPECT in persistent post-concussion syndrome after mild head injury: Comparison with MRI/CT. Brain Inj. 11, $115-124$.

King, N.S., Crawford, S., Wenden, F.J., Moss, N.E., and Wade, D.T. (1995). The Rivermead Post Concussion Symptoms Questionnaire: a measure of symptoms commonly experienced after head injury and its reliability. J. Neurol. 242, 587592.

Levin, H.S., Williams, D.H., Eisenberg, H.M., High Jr., W.H., and Guinto Jr., F.C. (1992). Serial MRI and neurobehavioural findings after mild to moderate closed head injury. J. Neurol. Neurosurg. Psychiatry 55, 255-262.

Lewine, J.D., Davis, J.T., Bigler, E.D., Thoma, R., Hill, D., Funke, M., Sloan, J.H., Hall, S., and Orrison, W.W. (2007). Objective documentation of traumatic brain injury subsequent to mild head trauma: multimodal brain imaging with MEG, SPECT, and MRI. J. Head Trauma Rehabil. 22, 141-155. 
Lowenstein, D.H., Thomas, M.J., Smith, D.H., and McIntosh, T.K. (1992). Selective vulnerability of dentate hilar neurons following traumatic brain injury: a potential mechanistic link between head trauma and disorders of the hippocampus. J. Neuroscience 12, 4846-4853.

Lyeth, B.G., Jenkins, L.W., Hamm, R.J., Dixon, C.E., Phillips, L.L., Clifton, G.L., Young, H.F., and Hayes, R.L. (1990). Prolonged memory impairment in the absence of hippocampal cell death following traumatic brain injury in the rat. Brain Res. 526, 249-258.

Lorberboym, M., Lampl, Y., Gerzon, I., and Sadeh, M. (2002). Brain SPECT evaluation of amnestic ED patients after mild head trauma. Am. J. Emerg. Med. 20, 310-313.

Maldjian, J.A., Laurienti, P.J., Kraft, R.A., and Burdette, J.H. (2003). An automated method for neuroanatomic and cytoarchitectonic atlas-based interrogation of fMRI data sets. Neuroimage 19, 1233-1239.

Marshall, L.F., Eisenberg, H.M., Jane, J.A., Luerssen, T.G., Marmarou, A., and Foulkes, M.A. (1991). A new classification of head injury based on computerized tomography. J. Neurosurg. 75, s14-s20.

McAllister, T.W., Saykin, A.J., Flashman, L.A., Sparling, M.B., Johnson, S.C., Guerin, S.J., Mamourian, A.C., Weaver, J.B., and Yanofsky, N. (1999). Brain activation during working memory 1 month after mild traumatic brain injury: a functional MRI study. Neurology 53, 1300-1308.

McAllister, T.W., Sparling, M.B., Flashman, L.A., Guerin, S.J., Mamourian, A.C., and Saykin, A.J. (2001). Differential working memory load effects after mild traumatic brain injury. NeuroImage 14, 1004-1012.

McCullagh, S., Oucherlony, D., Protzner, A., Blair, N., and Feinstein, A. (2001). Prediction of neuropsychiatric outcome following mild trauma brain injury: an examination of the Glasgow Coma Scale. Brain Inj. 15, 489-497.

Meyer-Lindenberg, A., Poline, J.B., Kohn, P.D., Holt, J.L., Egan, M.F., Weinberger, D.R., and Berman, K.F. (2001). Evidence for abnormal cortical functional connectivity during working memory in schizophrenia. Am. J. Psychiatry 158, 18091817.

Meythaler, J.M., Peduzzi, J.D., Eleftheriou, E., and Novack, T.A. (2001). Current concepts: diffuse axonal injury-associated traumatic brain injury. Arch. Phys. Med. Rehabil. 82, 1461-1471.

Nakayama, N., Okumura, A., Shinoda, J., Yasokawa, Y.T., Miwa, K., Yoshimura, S.I., and Iwama, T. (2006). Evidence for white matter disruption in traumatic brain injury without macroscopic lesions. J. Neurol. Neurosurg. Psychiatry 77, 850-855.

Olson, I.R., Page, K., Moore, K.S., Chatterjee, A., and Verfaellie, M. (2006). Working memory for conjunctions relies on the medial temporal lobe. J. Neuroscience 26, 4596-4601.

Owen, A.M., McMillan, K.M., Laird, A.R., and Bullmore, E. (2005). N-back working memory paradigm: a meta-analysis of normative functional neuroimaging studies. Hum. Brain Mapp. 25, 46-59.

Piekema, C., Kessels, R.P., Mars, R.B., Petersson, K.M., and Fernandez, G. (2006). The right hippocampus participates in short-term memory maintenance of object-location associations. Neuroimage 33, 374-382.

Pochon, J.B., Levy, R., Fossati, P., Lehericy, S., Poline, J.B., Pillon, B., Le Bihan, D., and Dubois B. (2002). The neural system that bridges reward and cognition in humans: an fMRI study. Proc. Natl. Acad. Sci. 99, 5669-5674.

Price, C.J., and Friston, K.J. (1999). Scanning patients with tasks they can perform. Hum. Brain Mapp. 8, 102-108.
Rizzo, P.A., Pierelli, F., Pozzessere, G., Floris, R., and Morocutti, C. (1983). Subjective posttraumatic syndrome. A comparison of visual and brain stem auditory evoked responses. Neuropsychobiology 9, 78-82.

Russell, W.R., and Smith, A. (1961). Post-traumatic amnesia in closed head injury. Arch. Neurol. 5, 4-17.

Rutgers, D.R., Toulgoat, F., Cazejust, J., Fillard, P., Lasjaunias, P., and Ducreux, D. (2008). White matter abnormalities in mild traumatic brain injury: a diffusion tensor imaging study. Am. J. Neuroradiol. 29, 514-519.

Sadowski-Cron, C., Schneider, J., Senn, P., Radanov, B.P., Ballinari, P., and Zimmermann, H. (2006). Patients with mild traumatic brain injury: immediate and long-term outcome compared to intra-cranial injuries on CT scan. Brain Inj. 20, 1131-1137.

Salmond, C.H., Chatfield, D.A., Menon, D.K., Pickard, J.D., and Sahakian, B.J. (2005). Cognitive sequelae of head injury: Involvement of basal forebrain and associated structures. Brain $128,189-200$.

Schmahmann, J.D., and Pandya, D.N. (1997). The cerebrocerebellar system. Int. Rev. Neurobiol. 41, 31-60.

Schmand, B., Bakker, D., Saan, R., and Louman, J. (1991). [The Dutch Reading Test for Adults: a measure of premorbid intelligence level]. Tijdschr. Gerontol. Geriatr. 22, 15-19.

Schoenhuber, R., Bortolotti, P., Malavasi, P., Marzolini, S., Tonelli, L., and Merli, G.A. (1983). Brain stem auditory evoked potentials in early evaluation of cerebral concussion. J. Neurosurg. Sci. 27, 157-159.

Shaw, N.A. (2002). The neurophysiology of concussion. Prog. Neurobiol. 67, 281-344.

Slemmer, J.E., Matser, E.J., De Zeeuw, C.I., and Weber, J.T. (2002). Repeated mild injury causes cumulative damage to hippocampal cells. Brain 125, 2699-2709.

Smits, M., Dippel, D.W., Houston, G.C., Wielopolski, P.A., Koudstaal, P.J., Hunink, M.G., and Van der Lugt, A. (2009). Postconcussion syndrome after minor head injury: brain activation of working memory and attention. Hum. Brain Mapp. 30, 2789-2803.

Snaphaan, L., Rijpkema, M., van Uden, I., Fernández, G., and de Leeuw, F.E. (2009). Reduced medial temporal lobe functionality in stroke patients: a functional magnetic resonance imaging study. Brain 132, 1882-1888.

Soustiel, J.F., Hafner, H., Chistyakov, A.V., Barzilai, A., and Feinsod, M. (2005). Trigeminal and auditory evoked responses in minor head injuries and post-concussion syndrome. Brain Inj. 9, 805-813.

Spielberger, C.D. (1983). Manual for the State-Trait Anxiety Inventory. Consulting Psychologists Press: Palo Alto, CA.

Squire, L.R., and Zola-Morgan, S. (1991). The medial temporal lobe memory system. Science 253, 1380-1386.

Stroop, J.R. (1935). Studies of interference in serial verbal reactions. J. Exp. Psychol. 18, 643-662.

Stulemeijer, M., Vos, P.E., and Van der Werf, S.P. (2007). Cognitive complaints after mild traumatic brain injury: things are not always what they seem. J. Psychosomat. Res. 63, 637-645.

Sundin, E.C., and Horowitz, M.J. (2002). Impact of Event Scale: psychometric properties. Br. J. Psychiatry 180, 205-209.

Ulug, A.M., Moore, D.F., Bojko, A.S., and Zimmerman, R.D. (1999). Clinical use of diffusion-tensor imaging for diseases causing neuronal and axonal damage. Am. J. Neuroradiol. 20, 1044-1048.

Umile, E.M., Sandel, M.E., Alavi, A., Terry, C.M., and Plotkin, R.C. (2002). Dynamic imaging in mild traumatic brain injury: 
Support for the theory of medial temporal vulnerability. Arch. Phys. Med. Rehabil. 83, 1506-1513.

Van der Naalt, J., Van Zomeren, A.H., Sluiter, W.J., and Minderhoud, J.M. (1999). One year outcome in mild to moderate head injury: the predictive value of acute injury characteristics related to complaints and return to work. J. Neurol. Neurosurg. Psychiatry 66, 207-213.

Von Wowern, F. (1966). Posttraumatic amnesia and confusion as an index of severity in head injury. Acta. Neurol. Scand. 42, 373-378.

Vos, P.E., Battistin, L., Birbamer, G., Gerstenbrand, F., Potapov, A., Prevec, T., Stephan, Ch.A., Traubner, P., Twijnstra, A., Vecsei, L., and von Wild, K. (2002). EFNS guideline on mild traumatic brain injury: report of an EFNS task force. Eur. J. Neurol. 9, 207-219.

Watson, M.R., Fenton, G.W., McClelland, R.J., Lumsden, J., Headley, M., and Rutherford, W.H. (1995). The post-concussional state: neurophysiological aspects. Br. J. Psychiatry 167, 514-521.

Wechsler, D. (1997a). Wechsler Adult Intelligence Scale-Third Edition (WAIS-III). Psychological Corporation: San Antonio, TX.
Wechsler, D. (1997b). Wechsler Memory Scale-III-Third Edition. Psychological Corporation: San Antonio, TX.

Weis, S., Klaver, P., Reul, J., Elger, C.E., and Fernandez, G. (2004). Temporal and cerebellar brain regions that support both declarative memory formation and retrieval. Cereb. Cortex 14, 256-267.

Zakzanis, K.K. (2001). Statistics to tell the truth, the whole truth, and nothing but the truth: formulae, illustrative numerical examples, and heuristic interpretation of effect size analyses for neuropsychological researchers. Arch. Clin. Neuropsychol. $16,653-667$.

Address correspondence to: Pieter E. Vos, M.D., Ph.D. Department of Neurology (935) Radboud University Nijmegen Medical Centre P.O. Box 9101, $6500 \mathrm{HB}$ Nijmegen, The Netherlands E-mail: p.vos@neuro.umcn.nl 
